\title{
Coma hiponatrêmico como manifestação de doença de Addison
}

\author{
Hyponatremic coma as a manifestation of Addison's disease
}

\author{
Paulo S.M. Esperon*
}

\section{Resumo}

Objetivo: alertar sobre a importância de distúrbio hidroeletrolítico grave devido a doença crônica não previamente diagnosticada.

Método: relato de um caso de coma hiponatrêmico devido a crise adrenal em criança portadora de doença de Addison não diagnosticada previamente, apesar de história clínica sugestiva desta doença há cerca de quatro anos. Em revisão bibliográfica, baseada nos sistemas Medline e Lilacs, não foi encontrado relato de evolução com duração tão prolongada entre as manifestações iniciais da doença de Addison e seu diagnóstico.

Resultado: após atendimento inicial à emergência, com correção da hiponatremia grave com hipovolemia discreta, hipernatriúria e hiperpotassemia, o diagnóstico diferencial deste distúrbio hidroeletrolítico revelou tratar-se de uma crise adrenal secundária a uma insuficiência adrenal primária crônica (doença de Addison). $\mathrm{O}$ tratamento com hidrocortisona oral possibilitou alta hospitalar com correção total dos distúrbios metabólicos.

Conclusão: é importante manter um alto grau de suspeita de doença de Addison para evitar as graves conseqüências de seu diagnóstico tardio, como distúrbios hidroeletrolíticos graves, além de atraso de crescimento e desenvolvimento.

J Pediatr (Rio J) 2001; 77 (4): 337-40: doença de Addison, hiponatremia, coma hiponatrêmico, crise adrenal, insuficiência adrenal.

\section{Introdução}

A doença de Addison é a causa mais comum de insuficiência adrenal primária crônica, sendo, na atualidade, mais freqüentemente causada por destruição auto-imune progressiva do córtex adrenal (adrenalite auto-imune) e tendo uma prevalência de 39 a 60 casos por milhão na população geral $^{1,2}$.

A maioria dos sinais e sintomas de insuficiência adrenal são inespecíficos e costumam manifestar-se de forma insi-

* Pediatra especialista em Terapia Intensiva Pediátrica pela AMIB-SBP. Ex-Médico chefe da UTIP do Hospital Bruno Born - Lajeado, RS.

\begin{abstract}
Objective: to show the importance of severe hydroelectrolytic disorder as a consequence of previously undiagnosed chronic disease.

Description: case report of hyponatremic coma caused by adrenal crisis in a child with previously undiagnosed Addison's disease despite suggestive clinical findings of this disease in the last four years. After correction of severe hyponatremia, mild hypovolemia, hypernatriuria and hyperkalemia, the differential diagnosis of this hydroelectrolytic disorder revealed an adrenal crisis resulting from a primary chronic adrenal insufficiency. Oral treatment with hydrocortisone was efficient in correcting the metabolic disorder and the patient was then discharged.

Comments: it is very important to highly suspect of Addison's disease in order to avoid the dangerous consequences of late diagnosis such as severe hydroelectrolytic disorders and retarded growth and development.
\end{abstract}

J Pediatr (Rio J) 2001; 77 (4): 337-40: Addison's disease; hyponatremia; hyponatremic coma; adrenal crisis; adrenal insufficiency.

diosa $^{1,2}$. A hiponatremia é um achado laboratorial freqüente de insuficiência adrenal e, quando de instalação rápida e severidade suficiente, pode causar convulsões e coma. As causas de hiponatremia são variadas e, no seu diagnóstico diferencial, deve levar-se em conta a volemia e a natriurese dos pacientes. A hiponatremia da insuficiência adrenal cursa com hipovolemia e hipernatriurese ${ }^{3}$. A crise adrenal aguda é mais freqüentemente desencadeada por infecção. Não é comum o diagnóstico da doença de Addison ser feito através de apresentação na forma de coma por hiponatremia severa. 
O autor descreve um caso de doença de Addison, diagnosticado através de crise adrenal aguda e coma hiponatrêmico. Em razão da história pregressa e do grau acentuado de desnutrição, é provável que a insuficiência adrenal tenha se instalado há muito tempo. Foi feita uma breve revisão da literatura e não foram encontrados, nas bases de dados pesquisadas, relatos de outros casos de doença de Addison com esta forma de apresentação. Discute-se, também, a importância da valorização da sintomatologia e do diagnóstico precoce.

\section{Relato do caso}

Menino de 9 anos, branco, pais agricultores, procedentes do meio rural de pequena cidade da região do vale do Taquari, no interior do RS. Foi admitido na UTIP do Hospital Bruno Born (Lajeado, RS) por crise convulsiva seguida de coma desde a noite anterior à internação. Nasceu de parto vaginal, normal, a termo, pesando $2.000 \mathrm{~g}$ e sem intercorrências neonatais. Pais e irmã normais.

Há aproximadamente 4 anos, iniciou com episódios freqüentes de vômitos e pneumonias de repetição. Anorexia, astenia, adinamia importantes e emagrecimento acentuado, ficando muitas vezes até 15 dias sem forças para levantar-se da cama. Alto grau de absenteísmo escolar, com rendimento muito baixo no aprendizado. Relato de extrema dificuldade para tolerar baixas temperaturas ambientais. Trazia resultados de investigações realizadas em dois Hospitais Universitários na capital do estado, nos últimos 3 anos, que concluíram por refluxo gastroesofágico e bronquite crônica. Veio transferido do Hospital de sua cidade, onde estava internado nos últimos 25 dias, recebendo medicação antibiótica endovenosa para tratamento de pneumonia.

Exame físico: peso de $16 \mathrm{~kg}(<\mathrm{P} 3)$, estatura de $1,0 \mathrm{~m}$ (<P3), afebril, desidratado de I grau, aspecto de desnutrição severa e em coma com hiper-extensão dos membros, sem rigidez de nuca e com pupilas isocóricas e reagentes à luz. Mucosas coradas e pele escura. Ausculta cardíaca normal e pulmonar com diminuição de murmúrio vesicular à esquerda. Sem anormalidades ao exame abdominal. Genitália masculina com testículos de tamanho normal para a idade. Extremidades frias, pulsos femurais simétricos e amplos.
Exames laboratoriais: hemograma com hemoglobina de $11,5 \mathrm{~g} / \mathrm{dl}$, hematócrito de $35 \%$, com 8700 leucócitos/dl com $1 \%$ de eosinófilos. Gasometria arterial com $\mathrm{pH}-7,23$ e bicarbonato de $24 \mathrm{mEq} / \mathrm{l}$. Sódio sérico de $102 \mathrm{mEq} / \mathrm{l}$, potássio sérico de 5,1 mEq/l, Ca iônico sérico de 1,02 $\mathrm{mMol} / \mathrm{l}$, glicemia de $186 \mathrm{mg} / \mathrm{dl}$, Uréia plasmática de 16 $\mathrm{mg} / \mathrm{dl}$, Creatinina plasmática de $0,8 \mathrm{mg} / \mathrm{dl}$, sódio urinário de $74 \mathrm{mEq} / \mathrm{l}$ e potássio urinário de24,7 mEq/l (amostra isolada).

Rx de tórax com consolidação parenquimatosa extensa à esquerda.

Foi iniciado tratamento antibiótico com ampicilina EV. A correção inicial rápida do sódio sérico foi feita com $\mathrm{NaCl}$ a $3 \%$ em 6 horas e foi calculada correção lenta para 125 $\mathrm{mEq} / \mathrm{l} \mathrm{em} 48$ horas (este valor foi atingido apenas no $5^{\circ} \mathrm{dia}$ de internação). A Tabela 1 mostra a evolução dos valores do sódio e potássio séricos.

Em função da desnutrição acentuada, hiponatremia severa com sódio urinário alto e pele escura, foi feita hipótese de doença de Addison e solicitada dosagem de cortisol sérico. O paciente saiu do coma logo após ter atingido natremia acima de $110 \mathrm{mEq} / \mathrm{l}$ e começou a ser realimentado progressivamente. Mantinha tendência constante à hiponatremia. No segundo dia de internação foi recebido o resultado do cortisol sérico: < que $1,2 \mathrm{mcg} / \mathrm{dl}$ às $8 \mathrm{~h}$ e < que 1 $\mathrm{mcg} / \mathrm{dl}$ às $16 \mathrm{~h}$ (valores de referência em mcg/dl: $8 \mathrm{~h}=5-23$; $16 \mathrm{~h}=3-15)$. As dosagens de ACTH e anticorpos antiadrenais foram de $2900 \mathrm{pg} / \mathrm{ml}$ (normal até $46 \mathrm{pg} / \mathrm{ml}$ ) e $<1: 2$ (normal < 1: 2) respectivamente.

Rx e tomografia computadorizada do abdome mostraram adrenais normais e sem sinais de calcificações.

Com esses dados, confirmou-se a hipótese inicial de crise adrenal aguda desencadeada por pneumonia e secundária à doença de Addison. Iniciou-se reposição hormonal com hidrocortisona na dose de $15 \mathrm{mg} / \mathrm{m}^{2}$ via oral (2/3 às $8 \mathrm{~h}$ e $1 / 3$ entre 16 h e $18 \mathrm{~h}$ ), havendo normalização definitiva do sódio sérico.

O paciente recebeu alta hospitalar 14 dias após a internação, pesando $17 \mathrm{~kg}$, recebendo hidrocortisona oral, orientado a retornar para consulta ambulatorial em 2 semanas.

Tabela 1 - Evolução dos valores de sódio e potássio séricos

\begin{tabular}{lcccccccc}
\hline & Internação & $\mathbf{1}^{\mathbf{0}}$ dia & $\mathbf{2}^{\mathbf{0}}$ dia & $\mathbf{3}^{\mathbf{0}}$ dia & $\mathbf{4}^{\mathbf{0}}$ dia & $\mathbf{5}^{\mathbf{0}}$ dia & $\mathbf{6}^{\mathbf{0}}$ dia & alta \\
\hline sódio $(\mathrm{mEq} / \mathrm{l})$ & 102 & 105 & 114 & 115 & 113 & 125 & 137 & 142 \\
Potássio $(\mathrm{mEq} / \mathrm{l}$ & 5,1 & 5,1 & 4,8 & 3,6 & 3,2 & 4,5 & 3,9 & 4,5 \\
\hline
\end{tabular}




\section{Discussão}

A produção insuficiente de hormônios adrenais pode ocorrer como resultado de doenças do córtex adrenal (insuficiência adrenal primária), ou do sistema hipotalâmicopituitário por secreção deficiente de hormônio liberador de corticotrofina (insuficiência adrenal central) ou do hormônio adrenocorticotrófico (insuficiência adrenal secundária $)^{4}$.

A insuficiência adrenal primária pode ser congênita ou adquirida. As formas congênitas são bastante raras e costumam manifestar-se no período neonatal ou nos primeiros meses de vida ${ }^{5-8}$, excetuando-se a adrenoleucodistrofia, que aparece no final da primeira década, mas cursa com graves alterações neurológicas como cegueira, surdez, demência, quadriparesia e morte ${ }^{9}$.

As formas adquiridas de insuficiência adrenal primária têm como modelo básico a doença de Addison que, originalmente, tinha a tuberculose como causa mais comum. Atualmente, a maior parte dos casos de doença de Addison estão relacionados à destruição auto-imune das adrenais (adrenalite auto-imune) ou são idiopáticos ${ }^{10}$. O sinal mais específico de insuficiência adrenal primária crônica é a hiperpigmentação da pele e mucosas em decorrência dos altos níveis de hormônio adrenocorticotrófico (ACTH) circulantes. Os achados de laboratório costumam ser hiponatremia (freqüente), hiperpotassemia, acidose e discreta elevação da creatinina sérica. O diagnóstico é confirmado pela presença de valores séricos muito baixos de cortisol, combinados com altos níveis de ACTH, além de ausência de resposta ao teste de estimulação com $\mathrm{ACTH}^{11}$.

Ainda são relatados dois outros tipos de insuficiência adrenal primária adquirida que também possuem uma natureza auto-imune:

1) síndrome auto-imune poliglandular tipo I, que ocorre no início da vida e associa-se com candidíase mucocutânea, hipoparatireoidismo e, pouco freqüentemente, com diabetes melito insulino-dependente ou com insuficência tireoidiana;

2) síndrome auto-imune poliglandular tipo II que ocorre mais tardiamente e está associada com doença tireoidiana, diabete melito insulino-dependente ou com os dois ao mesmo tempo, mas sem hipoparatireoidismo ${ }^{12}$.

A insuficiência adrenal primária adquirida pode também estar relacionada com infecção por HIV ou por infecções oportunistas a ele associadas ${ }^{13}$, assim como com muitos outros microorganismos e com certas drogas ${ }^{4}$.

O paciente apresentado neste caso manifestou, durante aproximadamente 4 anos, sinais e sintomas bastante sugestivos, embora inespecíficos, de insuficiência adrenal como náuseas, vômitos, anorexia, astenia, perda acentuada de peso e dificuldade em tolerar temperaturas baixas.

Internou-se em coma sem sinais meníngeos e sem história de trauma de crânio. Não havia história familiar de epilepsia ou de crises convulsivas prévias no paciente. Não houve exposição a substâncias tóxicas, drogas ou medica- ções que tivessem possibilidade de causar convulsões e coma ou de precipitar uma crise adrenal aguda. Na nossa avaliação inicial, o dado que mais chamou atenção foi a coloração escura da pele do menino.

Com os dados de história, exame físico e resultados iniciais de laboratório, levantou-se a hipótese de crise adrenal aguda precipitada pela infecção pulmonare, provavelmente, secundária à insuficiência adrenal primária crônica (doença de Addison). Foi solicitada dosagem de cortisol sérico e, em vista do valor extremamente baixo encontrado, solicitou-se então dosagem de ACTH e iniciou-se a reposição hormonal com hidrocortisona.

$\mathrm{O}$ resultado bastante elevado do ACTH confirma, na prática, a hipótese inicial de insuficiência adrenal primária crônica, embora deva ser ressaltado o fato de não ter sido feito o teste de estimulação com ACTH, que seria o exame mais adequado para o diagnóstico definitivo ${ }^{4}$. Pelo fato de não termos possibilidade de prosseguir a investigação etiológica, decidiu-se encaminhar o paciente para complementação diagnóstica em outro serviço.

Paterson ${ }^{14}$ relata dois casos de atraso no diagnóstico da doença de Addison: um paciente de 20 anos de idade (4 dias até o diagnóstico) e outro de 70 anos (5 semanas até o diagnóstico, sendo que o paciente foi a óbito), concluindo que as manifestações clínicas iniciais de insuficiência adrenal são inespecíficas, e as anormalidades laboratoriais muitas vezes não são severas, o que poderia contribuir para atrasar o diagnóstico.

Em pacientes com insuficência adrenocortical, o rápido reconhecimento e o tratamento imediato, mesmo sem confirmação diagnóstica definitiva, são fundamentais e, muitas vezes, salvam a vida do paciente. Torna-se importante ter em mente que hiponatremia com sódio urinário elevado nem sempre indica a presença de síndrome da secreção inapropriada do hormônio anti-diurético, e a hipótese de doença de Addison deve ser sempre considerada, especialmente se houver hipercalemia associada ${ }^{15}$.

Deve também ser salientado que o achado de sódio sérico normal não exclui necessariamente o diagnóstico de doença de Addison, embora evidentemente o torne menos provável, constituindo-se em outro fator que pode contribuir para atrasar o diagnóstico ${ }^{16}$.

Na hipótese de realmente ter havido atraso no diagnóstico, este retardo pode ter trazido conseqüências graves para o crescimento e desenvolvimento deste paciente.

Fica o alerta para a necessidade de manter-se alto grau de suspeição quanto à possibilidade da presença de insuficiência adrenal em pacientes que se apresentem com hiponatremia, mesmo que não tenham hipercalemia concomitante, especialmente quando não há alterações clínicas e laboratoriais compatíveis com a associação de insuficiência renal. Nestas situações, o tratamento hormonal de emergência deve ser iniciado, mesmo sem confirmação laboratorial definitiva da deficiência de cortisol, baseado-se apenas nas dosagens anormais de eletrólitos séricos e urinários. 


\section{Referências bibliográficas:}

1. May ME, Vaughan ED, Carey RM. Adrenocortical insufficiency - clinical aspects. In: Vaughan ED Jr, Carey RM, eds. Adrenal disorders. New York: Thieme Medical; 1989. p.171-89.

2. Oelkers W, Diederich S, Bähr V. Recent advances in diagnosis and therapy of Addison's disease. In: Bhatt HR, James VHT, Besser GM, et al., eds. Advances in Thomas Addison's diseases. Vol 1. London: Journal of Endocrinology, Thomas Addison Society; 1994. p.69-80.

3. Berry PL, Belsha CW. Hyponatremia. Ped Clin North Am 1990; 37: 351-63.

4. Sperling MA. The adrenal cortex. In: Sperling MA, ed. Pediatric endocrinology. $1^{\text {st }}$ ed. Philadelphia: WB Saunders; 1996.p.301-4.

5. Sperling MA, Wolfsen AR, Fischer DA. Congenital adrenal hypoplasia. J Pediatr 1973; 81: 444.

6. Shepard TH, Landing BH, Mason DC. Familial Addison's disease: case report of two sisters with corticoid deficiency unassociated with hypoaldosteronism. J Dis Child 1959; 97:154.

7. Cheek DB, Perry JW. A salt wasting syndrome in infancy. Arch Dis Child 1958; 33:252.

8. Queloz JM, Capitanio MA, Kirkpatrick JA: Wolman disease. Radiology 1972; 104: 357-9.

9. Davis LE, Synder RD, Orth DN, et al. Adrenoleukodystrophy and adrenomyeloneuropathy associated with adrenal insufficiency in three generations of a kindred. Am J Med 1979; 66: 342-7.

10. Neufeld M, MacLaren NK, Blizzard RM. Autoimmune polyglandular syndrome. Pediatr Ann 1980; 9: 154-62.
11. Oelkers W. Adrenal insufficiency. N Engl J Med 1996; 335:1206-12.

12. Neufeld M, MacLaren NK, Blizzard RM. Two types of autoimmune Addison's disease associated with different polyglandular autoimmune (PGA) syndromes. Medicine 1981; 60:355-62.

13. Freda PU, Wardlaw SL, Brudney K, Goland RS. Clinical case seminar. Primary adrenal insufficiency in patients with the acquired immunodeficiency syndrome: a report of five cases. $\mathbf{J}$ Clin Endocrinol Metab 1994; 79: 1540-5.

14. Paterson JR, Neithercut WD, Spooner RJ. Delayed diagnosis of Addison's disease. Ann Clin Biochem 1990; 27: 378-81.

15. Waise A, Young RJ. Pitfalls in the management of acute adrenal insufficiency: discussion paper. J R Soc Med 1989; 82:741-2.

16. Janson A. A life threatening disease among children often diagnosed too late. Normal sodium levels do not exclude Addison's disease. Lakartidningen 2000; 97:470-1.

Endereço para correspondência:

Dr. Paulo Sérgio Merlo Esperon

Rua Tiradentes 190 - apto. 502 - Centro

CEP 95900-000 - Lajeado, RS

Fone: (51) 748.5848 - Fax: (51) 748.7100

E-mail: ps.esperon@bewnet.com.br 